\title{
Forces on a Sphere in the Presence of Static and Dynamic Roughness Elements
}

\author{
AK Norman*and BJ McKeon ${ }^{\dagger}$ \\ California Institute of Technology, Pasadena, CA 91125
}

\begin{abstract}
Though the effect of distributed roughness on flow over a sphere has been examined in detail, there have been few observations as to the effect of an isolated roughness element on the forces induced on a sphere that is in uniform flow. In this experimental study, we examine how the forces are altered due to both a stationary and dynamic three-dimensional roughness element in the Reynolds number range of $5 \times 10^{4}$ to $5 \times 10^{5}$. It is found that even a small change to the geometry of the sphere, by adding a cylindrical roughness element with a width and height of $1 \%$ the sphere diameter, dramatically alters the drag and lateral forces over a wide range of Reynolds numbers. Of particular interest is that the mean of the lateral force magnitude can be increased by a factor of about seven, compared with a stationary stud, by moving the isolated roughness at a constant angular velocity about the sphere. These results can be applied to tripping a laminar boundary layer, steering a bluff body, and increasing the mixing of two fluids, using a minimal amount of energy input. This research is a first step towards understanding the interaction between time dependent surface motion and the subsequent alteration of the location of the boundary layer separation line and wake development.
\end{abstract}

\section{Introduction}

The sphere is a bluff body that is simple due to its symmetry, but at the same time the flow field is complex owing to the large separated region and variable separation line. The unsteady forces on a sphere have been observed since the time of Newton, ${ }^{1}$ and their exact nature continues to remain elusive.

For sphere Reynolds numbers (where $R e=\rho U_{\infty} D / \mu$ is based on sphere diameter $D$, free stream velocity $U_{\infty}$, density $\rho$, and dynamic viscosity $\mu$ ) above approximately 800 and below the critical Reynolds number for transition of the sphere boundary layer, $R e_{\text {crit }} \approx 3 \times 10^{5}$, frequencies corresponding to both a small-scale shear layer instability and a larger wake instability are present in the wake, while below this lower limit only the larger scale is observed. ${ }^{2-5}$ With increasing Reynolds number the Strouhal number $\left(S t=f D / U_{\infty}\right.$, where $f$ is the frequency) of the shear instability increases $\operatorname{as}^{3} R e^{n}$, with $0.5 \leq n<1$ for $10^{3}<R e_{D}<10^{5}$, while the large-scale Strouhal number remains approximately equal to 0.2, typical of vortex shedding. In the subcritical regime, computational studies ${ }^{6,7}$ have also found low frequency oscillations of the wake, below that of the large-scale vortex shedding frequency.

For a smooth sphere, the force coefficient is a function of $R e$ by dimensional analysis: $C_{i}=\frac{F_{i}}{\frac{1}{2} \rho U_{\infty}^{2} \frac{\pi}{4} D^{2}}=$ $f(R e)$, where the relation is valid for $i$ corresponding to the drag force or one of the lateral forces. Though the mean drag force has been measured by many researchers, the literature concerning the dynamic forces on a sphere is very sparse. The subcritical Reynolds number range has been examined computationally by Yun et al. ${ }^{6}$ using large eddy simulation, while both the subcritical and supercritical regimes have been investigated by Constantinescu and Squires ${ }^{7}$ using detached eddy simulation. These computational studies had very few experimental force measurements to compare with. In the subcritical regime, Lauchle et al. ${ }^{8}$ indirectly measured the forces using an accelerometer, but only obtained the decay of the frequency spectrum for $S t>1.5$. For the supercritical regime, Willmarth and Enlow ${ }^{1}$ reported the frequency spectrum and the mean squared lift for the case of a rough sphere.

*Graduate Student, Graduate Aerospace Laboratories, Pasadena, CA, AIAA Student Member

${ }^{\dagger}$ Assistant Professor, Graduate Aerospace Laboratories, Pasadena, CA, AIAA Member 
Adding distributed roughness of height $k$ introduces another dimensionless parameter that alters the force coefficient: $C_{i}=f(R e, k / D)$. This case has been studied in detail by Achenbach, ${ }^{9}$ at least for mean quantities. The roughness causes the boundary layer to transition sooner, and thus the critical Reynolds number is lower.

If instead there is a single isolated roughness element (a "stud"), the streamwise angle, $\phi_{k}$ (see figure 2), of the element becomes important. Due to symmetry, the spanwise angle can be ignored. Taneda ${ }^{10}$ observed that placing a stud at $\phi_{k}=90^{\circ}$ changed the orientation of the supercritical wake. It is expected that the stud will cause local transition of the boundary layer and alter the separation line if the Reynolds number of the stud is large enough.

Finally, if the spanwise angle $\left.\theta_{k}\right)$ of the roughness varies with time such that the stud moves at constant angular frequency $\dot{\theta_{k}}$, the relation becomes

$$
C_{i}=f\left(R e, k / D, \phi_{k}, \dot{\theta_{k}} D / U_{\infty}\right)
$$

where the angular speed has been made dimensionless by dividing by $U_{\infty} / D$ (i.e. it is a type of Strouhal number). Here we will examine the influence of these extra dimensionless parameters to the sphere system with a view to exploring prescription of direction to the force vector using small dynamic changes to the surface roughness.

\section{Experimental Setup}

The experiments were performed in the $61 \mathrm{~cm} \times 61 \mathrm{~cm}$ recirculating wind tunnel at the California Institute of Technology, which has a free stream turbulence intensity of $\sqrt{\overline{u^{\prime} u^{\prime}}} / U_{\infty}<0.3 \%$, where $u^{\prime}(t)=u(t)-\bar{u}$ is the streamwise fluctuating velocity at a point, and $\bar{u}$ is the mean velocity at a point. The test section velocity range was between about $3 \mathrm{~m} / \mathrm{s}$ and $50 \mathrm{~m} / \mathrm{s}$, and the sphere was centered in the test section.

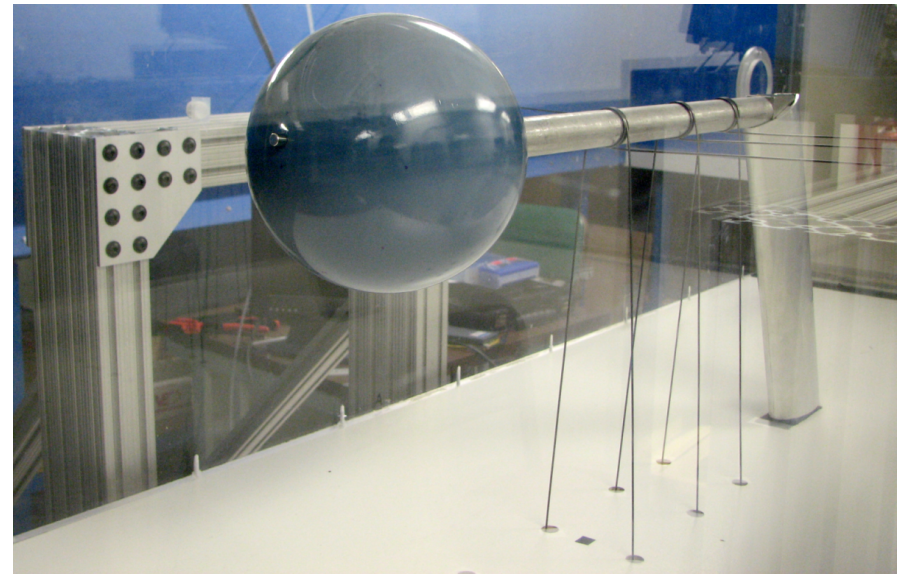

(a)

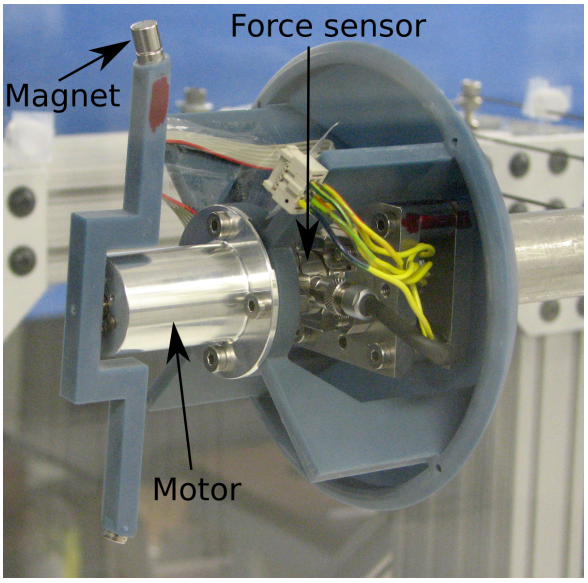

(b)

Figure 1: (a) Model in $0.61 \mathrm{~m}$ by $0.61 \mathrm{~m}$ recirculating wind tunnel test section, with piano wires used to increase the natural frequency of the sphere-support system. (b) Inside of the sphere showing the threecomponent force sensor and the motor which is used to drive the external stud.

\section{A. Model}

The experiment was designed to be able to take extremely accurate, time resolved force measurements, while supporting the sphere in a way that did not significantly affect the flow. Two hollow rapid-prototyped pieces were screwed together to form a sphere with a smooth seam, located at $\phi=120^{\circ}$, downstream of the supercritical separation point. The sphere was made using stereolithography, with a diameter of $D=15 \mathrm{~cm}$ and build tolerance of $\pm 0.002 D$, and was subsequently sanded to 600 grit to remove stair-stepping. The sphere was attached directly to a three-component piezoelectric force sensor (Kistler Type 9317B), which was itself attached to a stainless steel sting with an outside diameter of $2.54 \mathrm{~cm}$ and an inside diameter of 


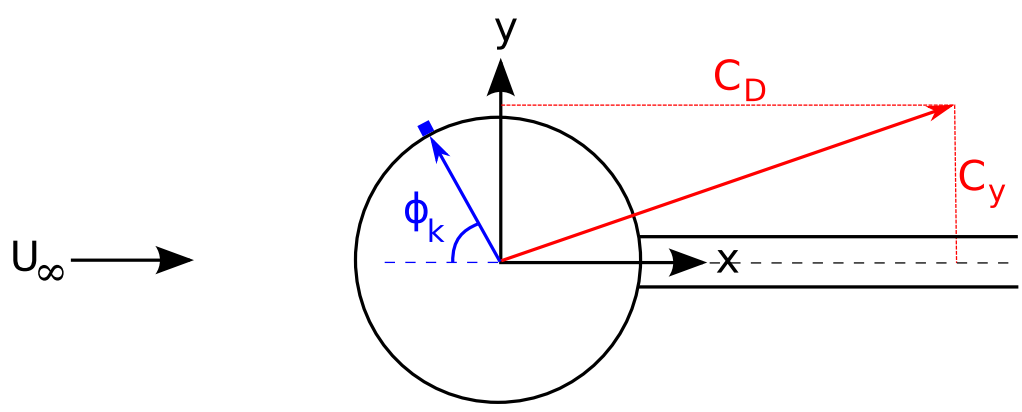

(a)

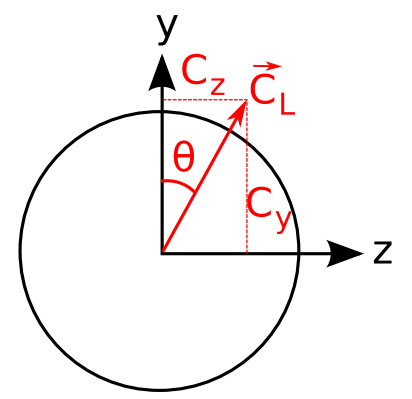

(b)

Figure 2: (a) Coordinate system chosen with $\mathrm{x}$ as the streamwise direction. $\phi$ is the streamwise angle from the stagnation point, with the $\mathrm{k}$ subscript indicating the streamwise angle to the stud. $C_{D}$ is the drag coefficient. (b) Looking downstream: the lateral force coefficient vector is labeled $\vec{C}_{L}$, and is composed of $C_{y} \hat{y}+C_{z} \hat{z} . \theta$ is the lateral angle from the y-axis to $\vec{C}_{L}$.

$1.27 \mathrm{~cm}$ (figure 1). This gave a sting to sphere diameter ratio of 0.17 , which should not significantly affect the flow for subcritical $R e$, but may lower the supercritical $C_{D} \cdot{ }^{11,12}$ The force sensor cables were passed through the sting, which was attached to a $5 \mathrm{~cm} \times 2.5 \mathrm{~cm}$ stainless steel beam. A streamlined fairing covered the beam to reduce blockage effects (see figure 1). Piano wires with a diameter of $0.1 \mathrm{~cm}$ were attached to the sting at three streamwise locations $(x / D=1.5,2.1$, and 2.6), which increased the natural frequency of the system from about $35 \mathrm{~Hz}$ to $75 \mathrm{~Hz}$. Each of the three piezo-elements was connected to a charge amplifier (Kistler 5010B), which output a voltage to a data acquisition board.

In order to produce a time-dependent surface roughness, a small motor and encoder were placed inside the sphere. The motor was attached to the force sensor on the same side as the sphere (figure 1b), so that there were no net forces measured due to the force of the stud on the sphere. The stud was a circular cylinder with a height and width of $1 \%$ the sphere diameter $(k / D=0.01)$. Its position was controlled by a magnet that was inside the sphere, which was moved around the sphere in the $\theta$-direction by the motor. The advantage of pulling the stud by using a magnet was that no holes or gaps needed to be machined in the sphere, allowing for a continuous, polished surface. The position of the stud was determined from a magnetic encoder attached to the motor, which produced 512 square wave pulses per revolution in two channels (to indicate the direction of rotation). The stud had a minimum rotational frequency of about $1 \mathrm{~Hz}$ (needed to overcome friction), and a maximum of about $7 \mathrm{~Hz}$, beyond which speed the stud would fly off of the sphere.

\section{B. Data Reduction}

The three-component piezoelectric force sensor allows time-resolved force measurements with a very low noise level on the order of $1 \mathrm{mN}$. However, true static measurements are impossible because the charge on a piezo-element drifts with time due to imperfect electrical insulation. This can be overcome by taking static measurements over a short amount of time, before the charge has drifted significantly. The static force results displayed later were obtained by taking measurements with the tunnel off, ramping the tunnel speed up, waiting 15 seconds, ramping down, and then estimating the drift with a linear fit to the zero flow data.

Once the mean force is found using the above method, the dynamic data for any given run condition is obtained by collecting data over an extended period of time (usually for $\hat{t}=t U_{\infty} / D=150000$ ), and the drift is estimated using a combination of the least squares method and cubic splines. If data is collected over a shorter period of time, a single polynomial curve is all that is needed to estimate the drift. Alternatively, using the high-pass filter that is built into the amplifier or using a high-pass filter during processing yielded very similar results. The cubic spline method was chosen due to its simplicity and zero-phase shift.

The natural frequency of the sphere-support system of about $75 \mathrm{~Hz}$ was well above the subcritical vortex shedding frequency. To isolate the signal from the structural vibrations, a low pass Butterworth filter was applied in both the forward and reverse directions (to have zero-phase shift) with a cut-off frequency of $50 \mathrm{~Hz}$. 


\section{Error Analysis}

The largest error was by far that caused by the (well known) sensitivity of sphere flow to the input boundary conditions. In other words, if the sphere was rotated, or moved with respect to the center of the test section, the mean forces were slightly altered. However, with a given setup the results were very repeatable, and the error caused by boundary conditions was minimal compared with the changes caused by the stud.

The linearity of the force sensor, as measured by the manufacturer, is $\leq \pm 44 \mathrm{mN}$ over the calibration range of $0-10 \mathrm{~N}$ for the three force components. The actual linearity is likely much better, as it is difficult to calibrate the sensors down to small loads, due to the charge drift. To verify the calibration, we placed small weights on the sensor and used the method described above to correct for the drift. The results were similar to those found by Schewe, ${ }^{13}$ who assumed that the sensor was linear over several decades. The cross-talk between components is $\leq \pm 2.2 \%$, as measured by the manufacturer.

\section{Notation}

The forces on a bluff body are dependent on time due to the unsteady wake. The force vector is given by $\vec{C}_{F}(\hat{t})=C_{D}(\hat{t}) \hat{x}+C_{y}(\hat{t}) \hat{y}+C_{z}(\hat{t}) \hat{z}$ (figure 2), where $\hat{t}=t U_{\infty} / D$ is the dimensionless time, and $\hat{x}, \hat{y}$, and $\hat{z}$ are unit vectors. The lateral force vector is defined to be $\vec{C}_{L}(\hat{t})=C_{y}(\hat{t}) \hat{y}+C_{z}(\hat{t}) \hat{z}$, with the lateral angle to $\vec{C}_{L}(\hat{t})$ being $\theta(\hat{t})$. The mean lateral force (which is equal to zero for symmetric flow) is given by $\vec{C}_{L}=\bar{C}_{y} \hat{y}+\bar{C}_{z} \hat{z}$, and the fluctuating forces are indicated by a prime, $C_{y}(\hat{t})=\bar{C}_{y}+C_{y}^{\prime}(\hat{t})$. The magnitude of the lateral force is $\left|\vec{C}_{L}(\hat{t})\right|=\sqrt{C_{y}(\hat{t})^{2}+C_{z}(\hat{t})^{2}}$, which is always greater than zero due to the unsteady flow.

An important quantity is the mean of the lateral force magnitude, $\overline{\left|\vec{C}_{L}(\hat{t})\right|}=\sqrt{C_{y}(\hat{t})^{2}+C_{z}(\hat{t})^{2}}$, because it shows the potential a control mechanism has for altering the lateral force.

\section{Results and Discussion}

\section{A. Stationary Roughness Element}

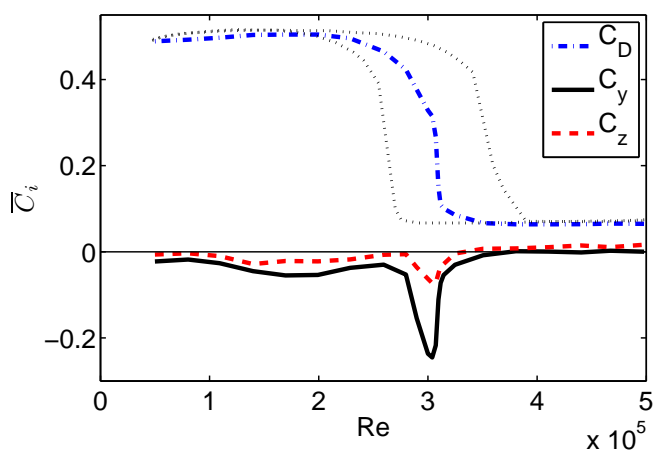

(a)

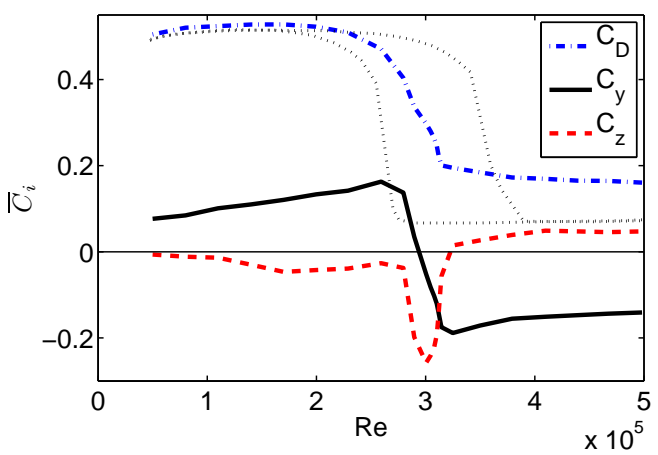

(b)

Figure 3: Mean force coefficients $\left(C_{i}\right.$, where $i$ represents the drag or one of the lateral force components) as a function of $R e$ without a stud (a) and with a $0.01 D$ stud placed at $\theta_{k}=0^{\circ}, \phi_{k}=70^{\circ}$ (b). $C_{D}$ falls between Achenbach's data ( $\cdots)$ for a smooth sphere (higher $\left.R e_{\text {crit }}\right)$ and slightly rough sphere $\left(k / D=2.5 \times 10^{-4}\right)$.

Consider the results for a single roughness element with characteristic size of $k / D=0.01$, positioned in the $+y$ direction, at $\theta_{k}=0^{\circ}$ and $\phi_{k}=70^{\circ}$. Figure 3a shows the classic drag curve, along with the two mean lateral force coefficients, $\bar{C}_{y}$ and $\bar{C}_{z}$, for the case of the smooth sphere. Our sphere is seen to have a critical Reynolds number between that of Achenbach's smooth sphere and slightly rough sphere, with the subcritical and supercritical drag coefficient in good agreement. No hysteresis was detected in the critical regime. The lateral forces are nearly zero, as expected due to symmetry, except for in the critical Re range. In this range the sphere is very sensitive to asymmetries because the boundary layer is beginning its transition to turbulence. Figure 3b shows the same results except with the small stud in place. Though the critical Re 


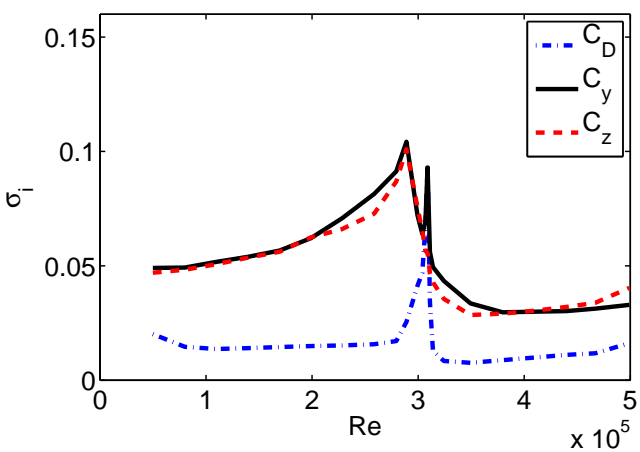

(a)

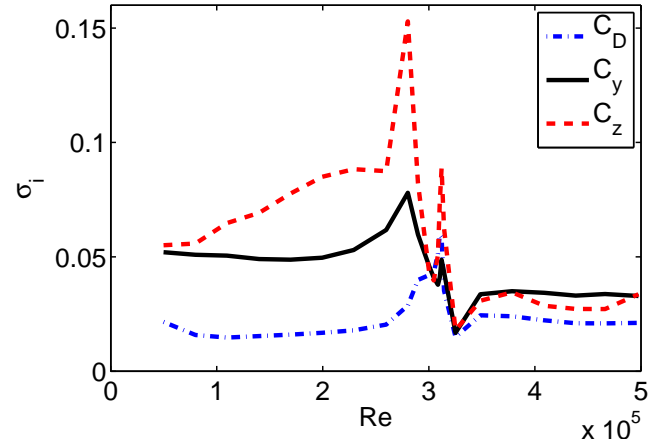

(b)

Figure 4: Standard deviation of the force coefficients as a function of $R e$ without a stud (a) and with a $0.01 D$ stud placed at $\theta_{k}=0^{\circ}, \phi_{k}=70^{\circ}(\mathrm{b})$.

is mostly unchanged, the supercritical drag is increased, and $\bar{C}_{y}$ now gradually increases with $R e$ until the critical regime, at which point the sign of the force changes. This phenomenon of the lateral force changing sign was observed by Hunt when examining the forces on a half roughened cricket ball. ${ }^{14}$ With the small stud, the magnitude of the lift to drag ratio is now approximately one in the supercritical regime. This indicates that the base flow has been significantly changed, which is a possible cause for the change in $\bar{C}_{z}$.

The standard deviation of the lateral forces is much larger than that of the drag, as seen in figure $4 a$. The lateral force fluctuations peak near the beginning of the critical regime, while the drag fluctuations peak near the end. When the stud is present, the subcritical lateral fluctuations become quite different, with the fluctuations in the plane of the stud being lower, and those in the opposite plane increasing (figure $4 \mathrm{~b}$ ). Another interesting feature is that, centered on $R e=3.2 \times 10^{5}$, the fluctuations are all small and of the same magnitude.

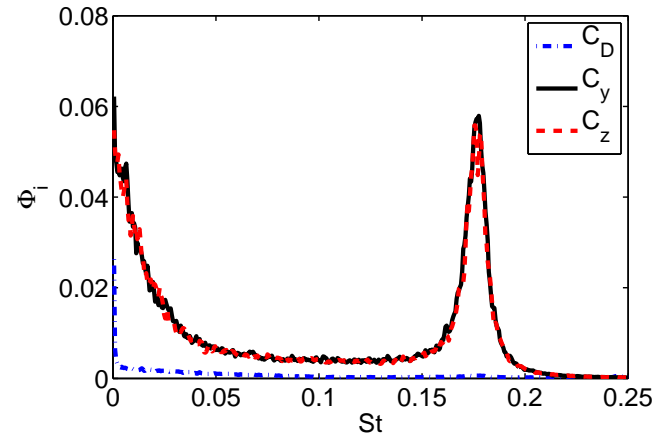

(a)

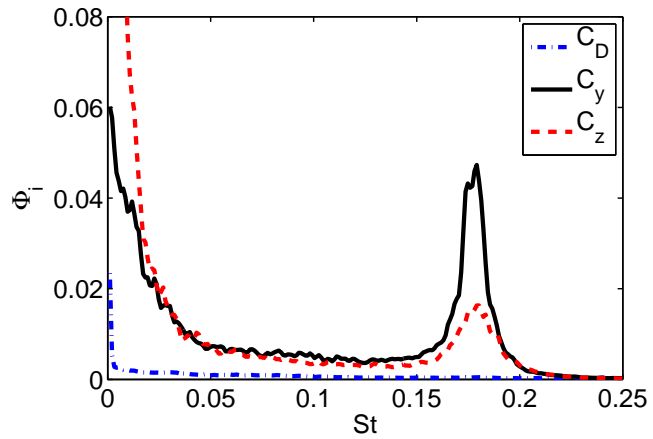

(b)

Figure 5: Subcritical Re of $8.0 \times 10^{4}$ showing the normalized spectral density of the dynamic forces without a stud (a) and with a $0.01 D$ stud placed at $\theta_{k}=0^{\circ}, \phi_{k}=70^{\circ}$ (b). The peak at a vortex shedding frequency of about $S t=0.18$ is clearly defined.

The power spectral density as a function of the dimensionless frequency is shown in figures 5 through 7 for a Reynolds number of $8.0 \times 10^{4}, 2.0 \times 10^{5}$, and $4.4 \times 10^{5}$, respectively. Each spectral component is normalized such that the total area under the spectrum equals the mean squared force fluctuations:

$$
\overline{C_{i}^{\prime 2}}=\int_{0}^{\infty} \Phi_{i}(S t) d(S t)
$$

where $\Phi_{i}$ is the power density of the $i^{t h}$ force component. A clear peak at the subcritical vortex shedding frequency is evident in figure $5 \mathrm{a}$, along with comparable energy at lower frequencies. When the stud is added 


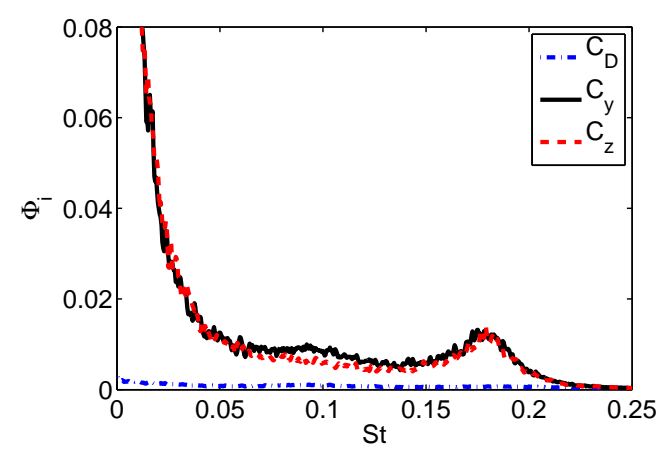

(a)

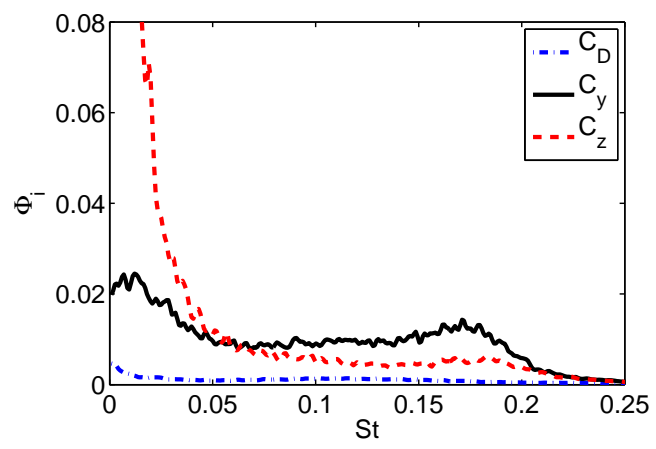

(b)

Figure 6: Subcritical Re of $2.0 \times 10^{5}$ showing the normalized spectral density of the dynamic forces without a stud (a) and with a $0.01 D$ stud placed at $\theta_{k}=0^{\circ}, \phi_{k}=70^{\circ}$ (b). The energy associated with the vortex shedding frequency is markedly reduced.

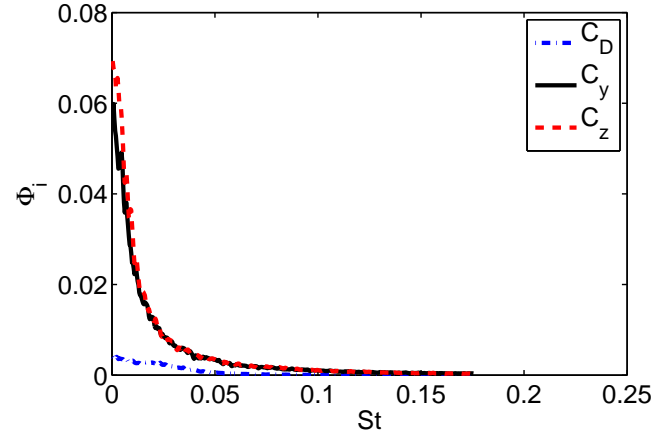

(a)

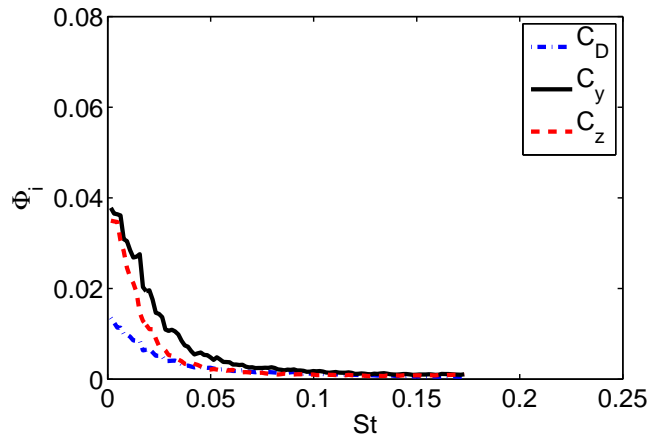

(b)

Figure 7: Supercritical Re of $4.4 \times 10^{5}$ showing the normalized spectral density of the dynamic forces without a stud (a) and with a $0.01 D$ stud placed at $\theta_{k}=0^{\circ}, \phi_{k}=70^{\circ}(\mathrm{b})$.

(again at $\phi_{k}=70^{\circ}$ in the $+y$ direction), the $C_{y}$ spectrum barely changes, while the vortex shedding peak greatly decreases in the other lateral direction (figure 5b), accompanied by more energy at lower frequencies. As the critical Reynolds number is approached, the peak in the spectrum at the vortex shedding frequency gradually disappears, until it is no longer detectable at $R e \cong 2.8 \times 10^{5}$. Figure 6 a shows the spectral density for an intermediate $R e$ of $2.0 \times 10^{5}$, where there is very little energy left that is associated with vortex shedding. Adding the stud (figure 6a) completely changes the spectrum, and the vortex shedding peak is essentially gone. For supercritical Re (figure 7a) there is no peak near the subcritical vortex shedding frequency of $S t=0.18$, for the smooth sphere case. For this Reynolds number, we were not able to measure beyond $S t \approx 0.2$ due to interference of the structural vibration peak. When the stud is added (figure $7 \mathrm{~b}$ ), the $C_{D}$ and $C_{y}$ spectrums overlap for $S t>0.4$.

Before examining the effect of a dynamic roughness element, the optimal position of a stationary stud needed to be found. Figure 8a shows the change in the lateral force coefficient as a function of the stud's streamwise angle and Reynolds number. Here, the stud is placed in the $+y$ direction, and the color indicates the magnitude of the mean lateral force with the smooth sphere values subtracted off: $\Delta \bar{C}_{y}=\bar{C}_{y}(w / s t u d)-$ $\bar{C}_{y}($ smooth $)$. It is expected that when the stud is near the front of the sphere it will not trip the boundary layer because the local Reynolds number of the stud is small, while for large $\phi_{k}$ there should also be little effect since the boundary layer has separated or already tripped. The results confirm this, showing that when the stud is near the front of the sphere $\left(\phi_{k} \lesssim 20^{\circ}\right.$ for subcritical, and $\phi_{k} \lesssim 30^{\circ}$ for supercritical), the lateral force is mostly unchanged. Similarly, when the stud is past the equator, beyond $100^{\circ}$, there is also little change. The stud has the largest effect between about 40 and 80 degrees. The maximum values occur 


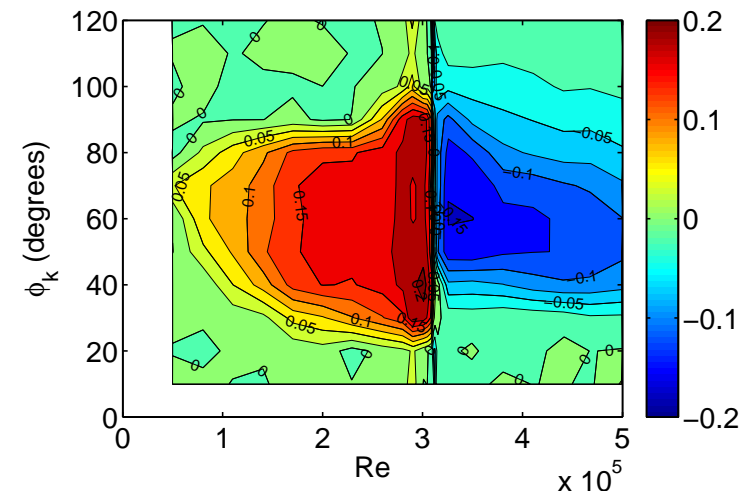

(a)

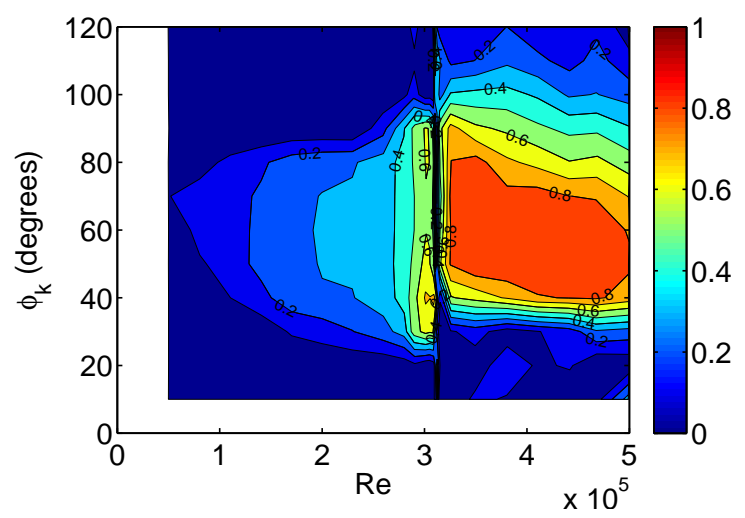

(b)

Figure 8: Contour plots of (a) $\Delta \bar{C}_{y}=\bar{C}_{y}(w / s t u d)-\bar{C}_{y}$ (smooth) and (b) $\Delta \bar{C}_{y} / \bar{C}_{D}$ as a function of the streamwise angle of the stud and $R e$, where the stud is placed in the $+y$ lateral direction $(\theta=0)$.

near the critical regime, and begin to drop off at lower and higher Re. Compared with the smooth sphere, the subcritical lateral force has a maximum of about $35 \%$ that of the subcritical drag coefficient of 0.5 , and the supercritical lateral force has a maximum of about $230 \%$ the supercritical drag. Note, however, that with the stud in place the drag also changes. Figure $8 \mathrm{~b}$ shows the absolute value of $\Delta \bar{C}_{y} / \bar{C}_{D}$, where $\bar{C}_{D}$ is the mean drag coefficient with the stud in place, which is a function of $R e$ and $\phi_{k}$. An interesting feature here is that the lift to drag ratio is almost equal to one over a large range of $\phi_{k}$ and $R e$ for supercritical Reynolds numbers. Thus, the mean forces can be dramatically altered by locally changing the shape of the body by only $1 \%$ of its diameter.

\section{B. Dynamic Roughness Element}

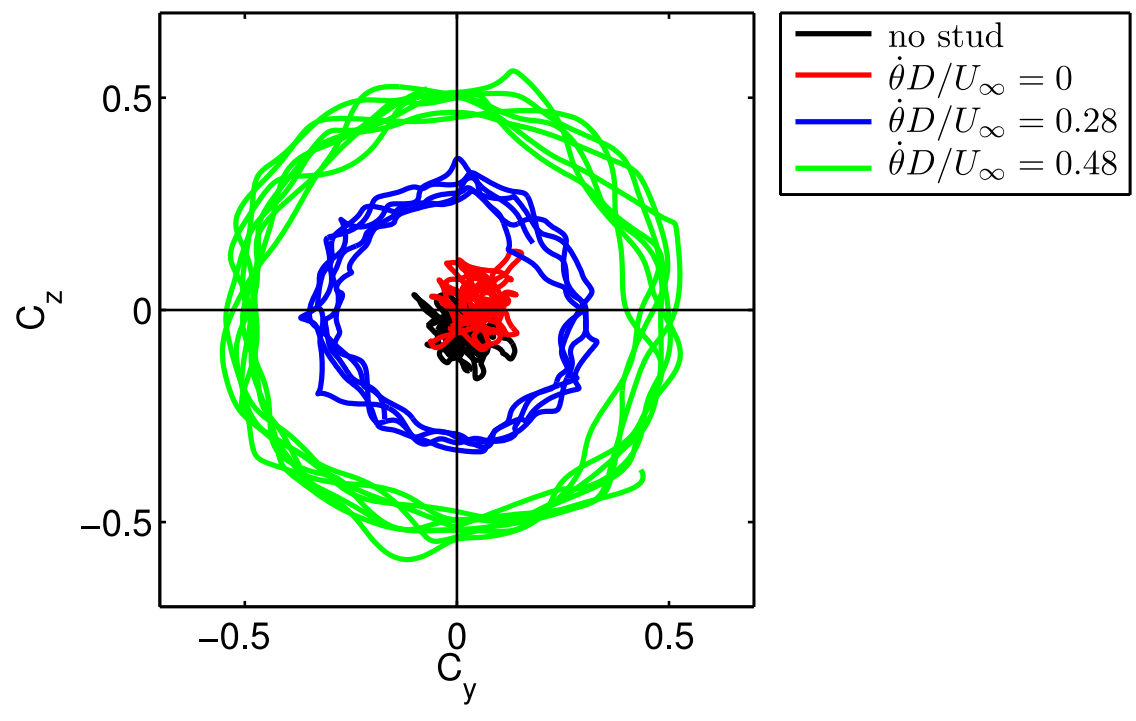

Figure 9: Time trace of the lateral force coefficients for $R e=8 \times 10^{4}$, showing that the dynamic stud completely changes the force characteristics. The mean of the lateral force magnitude of the moving stud is up to seven times larger than that of the stationary stud.

For the dynamic tests, the stud was placed at a streamwise angle of $70^{\circ}$, because the stud has the largest effect at that angle for low Reynolds numbers, which is important because when the stud is moving at its 
maximum angular frequency, the ratio $\dot{\theta} D / U_{\infty}$ is the largest for lower Re. For these experiments, a different sphere was used that had a slightly lower critical $R e$ of $2.8 \times 10^{5}$. Figure 9 shows a time trace of the lateral forces for a total time of $\hat{t}=100$ for the smooth sphere, a stationary stud at $\phi_{k}=70^{\circ}\left(\theta=0^{\circ}\right)$, and a dynamic stud moving at two different constant angular frequencies. The forces due to the stationary stud are very similar to the smooth sphere case, except with a small mean offset. By adding time dependence to the stud's position, the lateral forces are completely altered, with most of the spectral energy being near the frequency of stud rotation. The mean of the lateral force magnitude of the moving stud is up to seven times larger than that of the stationary stud. At any given instant the magnitude of the lateral force is approximately $\left|\vec{C}_{L}(\hat{t})\right|=0.5$ for $\dot{\theta} D / U_{\infty}=0.48$. This is surprisingly large considering that only a small stud is moving, yet when an entire golf ball spins about an axis perpendicular to its direction of motion at $\dot{\phi} D / U_{\infty}=0.48$, it has a mean lateral force of about $0.2 .{ }^{15}$ We suspect that the moving stud locally trips the boundary layer, and that it remains tripped for a period of time, causing transition over a wide spanwise area. Future experiments will oscillate the stud about a point in order to help verify this and determine if a similar mean lateral force can be achieved (because at any given time the dynamic stud produces a large lateral force, but the mean lateral force is zero).

As the stud moves around the sphere, the force lags behind the stud by an average angle $\theta_{\text {lag }}=\theta_{k}-\theta$ (figure 10). For the supercritical lag, $180^{\circ}$ is subtracted from $\theta_{\text {lag }}$, because the supercritical lateral force for the stationary stud is in the opposite direction of the subcritical lateral force. The general trend in figure 10 is that the force lag increases as the dimensionless parameter $\dot{\theta} D / U_{\infty}$ increases, indicating that this is a suitable parameter to collapse the data. Of particular interest is that at large angular frequencies, the force lags behind the stud by more than $180^{\circ}$. Figure 11 shows the mean of the lateral force magnitude $\left(\overline{\left|\vec{C}_{L}(\hat{t})\right|}=\overline{\sqrt{\left.C_{y}(\hat{t})^{2}+C_{z}(\hat{t})^{2}\right)}}\right.$ as a function of $\dot{\theta} D / U_{\infty}$. For the lowest Reynolds number case, the mean lateral force has a maximum. This could be due to tripping of more than half of the boundary layer at once. Future experiments will attempt to verify this by rotating the stud fast enough to trip the entire boundary layer and produce a supercritical flow.

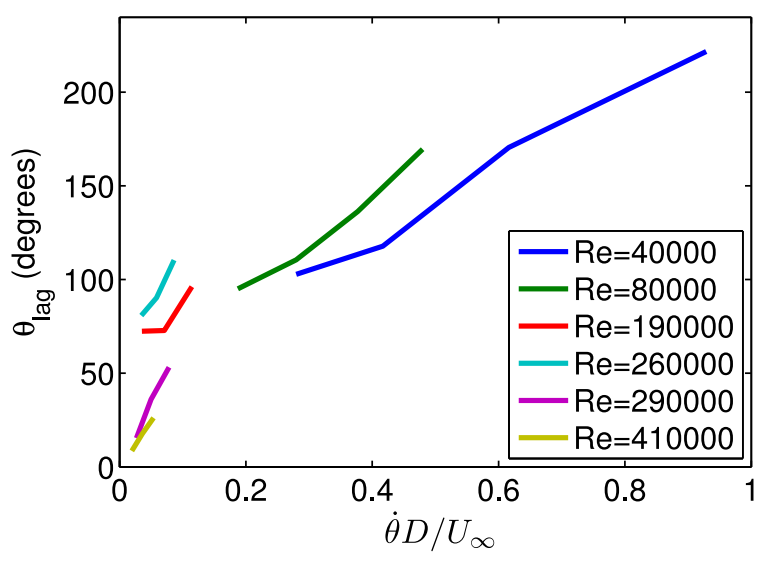

Figure 10: Angle that the lateral force vector lagged behind the stud $\left(\theta_{k}-\theta\right)$, as a function of dimensionless stud angular frequency.

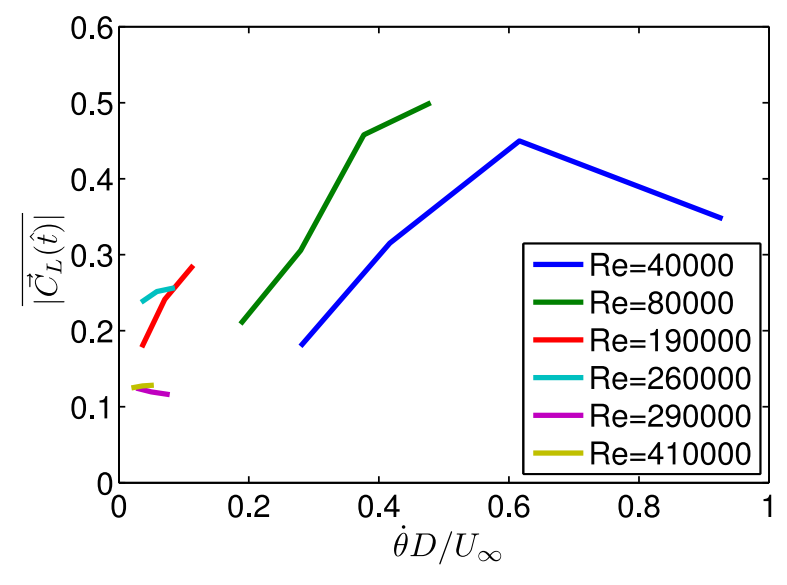

Figure 11: Mean of the lateral force magnitude as a function of dimensionless stud angular frequency.

\section{Conclusion}

In this experimental study we used a piezoelectric force sensor to obtain what we believe to be the first experimental measurements of the full spectral range of forces induced on a sphere by vortex shedding, and the forces caused by introducing both a static and dynamic isolated roughness element on the sphere surface, over a large range of Reynolds numbers.

For the smooth sphere, our results for the drag coefficient agree well with the literature. The mean lateral force was close to zero except for near the critical Reynolds number regime, where the flow is very sensitive to asymmetries on the sphere surface and other boundaries. The standard deviation of the forces 
reached a maximum near the critical $R e$, and the lift fluctuations were several times larger than the drag fluctuations for subcritical and supercritical $R e$. The spectral density for two representative $R e$ was shown, with good agreement between the two lateral forces. For the subcritical case, there is a distinct peak at the dimensionless frequency of $S t \cong 0.18$, corresponding to vortex shedding. In addition to this peak, there is also significant energy at lower frequencies. The spectral energy associated with vortex shedding gradually decreases as the critical Reynolds number is approached. For the supercritical case, there is no peak centered on the subcritical vortex shedding frequency.

When a small isolated roughness element with a characteristic length of $1 \%$ the sphere diameter is added at a streamwise angle between about $\phi_{k}=40^{\circ}$ and $80^{\circ}$, the forces are significantly changed. The lateral force component, that is in the same direction as the stud, increases with $R e$ until the critical $R e$, at which point the force direction reverses and slowly decreases with $R e$. The drag force is mostly unchanged for subcritical Re, but increases for supercritical. For $\phi_{k}=70^{\circ}$, the spectrum of the lateral force that is in the direction of the stud is only slightly changed, while the spectrum for the other lateral force has less energy near the vortex shedding frequency, and more at lower frequencies.

Dynamically moving the roughness element along the surface of the sphere at a fixed streamwise angle dramatically alters the forces. As the stud moves around the sphere, the lateral force vector follows the vector describing the stud location, with an average angle between the vectors that increases with increasing stud speed. For subcritical Re, the mean of the lateral force magnitude of the dynamic stud varies with dimensionless stud speed and was observed to have a maximum of about seven times that of the stationary stud. Future experiments will examine the connection between the force distribution and the flow separation line, along with the behavior of the wake.

\section{Acknowledgments}

Support from the National Science Foundation under Grant No. 0747672 (Program Manager William W. Schultz) is gratefully acknowledged.

\section{References}

${ }^{1}$ Willmarth, W. and Enlow, R., "Aerodynamic lift and moment fluctuations of a sphere," Journal of Fluid Mechanics, Vol. 36, No. 03, 1969, pp. 417-432.

${ }^{2}$ Sakamoto, H. and Haniu, H., "A study on vortex shedding from spheres in a uniform flow," ASME, Transactions, Journal of Fluids Engineering, Vol. 112, 1990, pp. 386-392.

${ }^{3}$ Kim, H. and Durbin, P., "Observations of the frequencies in a sphere wake and of drag increase by acoustic excitation," Physics of Fluids, Vol. 31, 1988, pp. 3260.

${ }^{4}$ Bakić, V. and Perić, M., "Visualization of flow around sphere for Reynolds numbers between 22000 and 400000 ," Thermophysics and Aeromechanics, Vol. 12, No. 3, 2005, pp. 307-315.

${ }^{5}$ Achenbach, E., "Vortex shedding from spheres," Journal of Fluid Mechanics, Vol. 62, No. 02, 1974, pp. 209-221.

${ }^{6}$ Yun, G., Kim, D., and Choi, H., "Vortical structures behind a sphere at subcritical Reynolds numbers," Physics of Fluids, Vol. 18, 2006, pp. 015102.

${ }^{7}$ Constantinescu, G. and Squires, K., "Numerical investigations of flow over a sphere in the subcritical and supercritical regimes," Physics of Fluids, Vol. 16, No. 5, 2004, pp. 1449.

${ }^{8}$ Lauchle, G. and Jones, A., "Unsteady Lift Force on a Towed Sphere," Journal of Fluids and Structures, Vol. 12, No. 8, 1998, pp. 949-958.

${ }^{9}$ Achenbach, E., "The effects of surface roughness and tunnel blockage on the flow past spheres," Journal of Fluid Mechanics, Vol. 65, No. 01, 1974, pp. 113-125.

${ }^{10}$ Taneda, S., "Visual observations of the flow past a sphere at Reynolds numbers between $10^{4}$ and $10^{6}$," Journal of Fluid Mechanics, Vol. 85, No. 01, 1978, pp. 187-192.

${ }^{11}$ Norman, A. and McKeon, B., "Effect of sting size on the wake of a sphere at subcritical Reynolds numbers," AIAA2008-4237, 2008.

${ }^{12}$ Hoerner, S., "Tests of Spheres With Reference to Reynolds Number, Turbulence, and Surface Roughness. Technical Memoradum No. 777," National Advisory Committee for Aeronautics, Langley, Virginia, 1935.

${ }^{13}$ Schewe, G., "A multicomponent balance consisting of piezoelectric force transducers for a high-pressure wind tunnel," Sensors and systems'82, 1982, pp. 13-23.

${ }^{14}$ Mehta, R., "Aerodynamics of Sports Balls," Annual Review of Fluid Mechanics, Vol. 17, No. 1, 1985, pp. 151-189.

${ }^{15}$ Bearman, P. and Harvey, J., "Golf ball aerodynamics," Aeronautical Quarterley, Vol. May, 1976, pp. 112-122. 